\title{
A Diffusion-Driven Model for Investigating Moisture Effects on Dielectric Response Measurement of Transformer Insulation
}

\author{
Yi Cui, St. Member, IEEE, Hui Ma, Member, IEEE, Tapan Saha, Senior Member, IEEE, Chandima Ekanayake, Member, \\ IEEE \\ School of Information Technology and Electrical Engineering, The University of Queensland \\ Brisbane, QLD, 4072, Australia \\ y.cui3@uq.edu.au,huima@itee.uq.edu.au, saha@itee.uq.edu.au, chandima@itee.uq.edu.au
}

\begin{abstract}
This paper proposes a diffusion-driven dielectric response model, which aims to reveal the effect of moisture diffusion on the dielectric response measurement of transformer insulation. In the proposed diffusion-driven model, the oilimpregnated pressboards are modeled as multiple layers and it is assumed moisture diffuses from one side of the pressboard. Then the dependency between the moisture concentration and dielectric losses and permittivity of the pressboard is studied. Subsequently, the dielectric response of the pressboard is calculated to model the dielectric relaxation behavior driven by the moisture migration between oil and pressboard. The proposed model is verified by laboratory experimental results.
\end{abstract}

Index Terms-Dielectric response, moisture diffusion, transformer insulation, loss factor (Tan $\delta$ ), dielectric dissipation

\section{INTRODUCTION}

Existence of moisture in a power transformer reduces the electrical strength of its oil-paper insulation system. It may also significantly accelerate the aging of the insulation in a transformer. Therefore, accurately evaluating the moisture contents inside a transformer tank has significant benefit for ensuring the reliable operation of a transformer.

Several researchers have investigated moisture diffusion process and proposed different methods to calculate diffusion coefficients of pressboard insulation based on experimental studies [1-10]. Ast [8] and $\mathrm{Du}$ [6] investigated moisture diffusion coefficients under different moisture concentrations and temperatures. Guidi and Fullerton [7] calculated diffusion coefficient by empirical curve-fitting from experimental data. Foss [11] investigated diffusion coefficients for nonimpregnated and oil-impregnated pressboards using experimental data from the literature. Howe [12] proposed a mathematical model to describe the moisture diffusion process by using finite difference method.

Although extensive studies have been carried out to model the moisture distribution in a transformer, proposed models were mainly based on the experiment and fitted data. So it is still a challenge for these techniques to accurately assess the moisture in a transformer, especially when the moisture is driven to migrate between oil and pressboard. In recent time, dielectric response method, i.e. frequency domain spectroscopy (FDS) measurement has been gaining popularity for estimating the moisture content [13]. It is therefore necessary to investigate the correlation between moisture diffusion process and dielectric response method to pave the way for an accurate moisture content estimation.

This paper aims to evaluate how the moisture diffusion between oil and pressboard may affect the dielectric response measurement. Finite element method is employed in this paper to calculate the moisture distribution in pressboard during the moisture diffusion process. Then a diffusion-driven model is proposed in which the pressboard is modelled as multiple layers of paper with different permittivity and dielectric loss (including both conductive and polarization loss). By using the proposed model, dielectric relaxation behaviour of pressboard due to moisture migration between oil and paper can be studied. Finally, dielectric response measurement (e.g. FDS) is performed on pressboard and measurement results are used to validate the proposed model.

\section{MOISTURE DIFFUSION OF OIL-PAPER INSULATION}

During diffusion process, the moisture distribution inside the pressboard is calculated by solving the Fick's second law.

$$
\frac{\partial C(x, t)}{\partial t}=\frac{\partial}{\partial x}\left(D \frac{\partial C(x, t)}{\partial x}\right)
$$

where $C(x, t)$ is the moisture content of pressboard at position $x$ and time $t . D$ denotes the diffusion coefficient. Here the diffusion coefficient has not been assumed as constant and it depends on both moisture concentration and temperature (2)

$$
D=D_{0} e^{\left[k\left[\bar{c}+E_{a}\left(\frac{1}{T_{0}}-\frac{1}{T}\right)\right]\right.}
$$

where $D_{0}=1.34 \times 10^{-13} \mathrm{~m}^{2} / \mathrm{s}$ and $k=0.5 . E_{a}$ is the activation energy which equals to $8074 \mathrm{~kJ} / \mathrm{mol}$ [2]. $T$ is the measured temperature in Kelvin . $T_{0}$ equals to $298 \mathrm{~K}$. 
The initial values and boundary condition for (1) is

$$
\begin{aligned}
& C(x, t=0)=C_{0}, C_{0}<0.5 \% \\
& C(x=0, t)=C_{s}, \frac{\partial C(x=l, t)}{\partial x}=0
\end{aligned}
$$

where $C_{0}$ is the moisture content in dry pressboards and initially assumed less than $0.5 \%$. $C_{s}$ is the constant source of moisture for diffusion.

\section{DIFFUSION-DRIVEN DIELECTRIC RESPONSE MODEL}

During the moisture diffusion process, dielectric response of pressboard can be influenced by non-equilibrium moisture distribution. To analyse the dielectric behaviour of pressboard with non-equilibrium moisture distribution, a diffusion-driven model is proposed in this paper (Fig. 1). In the proposed model, the pressboard with total thickness $l$ is sliced into finite layers of pressboard $(i=1,2, \ldots, \mathrm{N})$. Each layer of pressboard has different dielectric loss $\sigma_{i}$ and permittivity $\varepsilon_{i}$ due to the non-homogeneous distributed moisture.
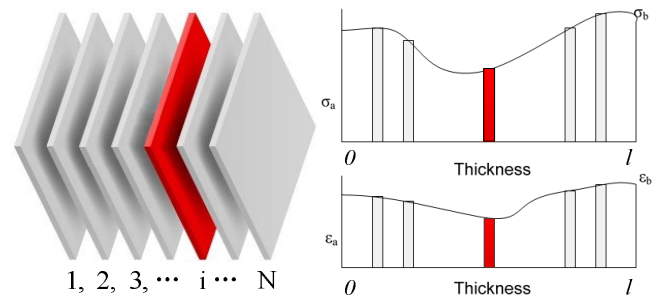

Fig. 1. Diffusion-driven model of pressboard

The impedance $Z(\omega)$ of the pressboard in frequency domain can be written as (4)

$$
Z(\omega)=\frac{1}{G(\omega)}=\sum_{i=1}^{N} Z_{i}(\omega)=\sum_{i=1}^{N} \frac{1}{G_{i}(\omega)}
$$

where $N$ denotes the total number of layers. $Z_{i}(\omega)$ and $G_{i}(\omega)$ are the impedance and admittance of the $i$-th layer of pressboard respectively. Based on the frequency domain dielectric spectroscopy theory [13], (4) can be rewritten as (5)

$$
Z^{*}(\omega)=\sum_{i=1}^{N} \frac{1}{\sigma_{i}+j \omega \varepsilon_{0} \varepsilon_{i}^{\prime}} \frac{\Delta x}{S}=\sum_{i=1}^{N} \frac{\sigma_{i}-j \omega \varepsilon_{0} \varepsilon_{i}^{\prime}}{\sigma_{i}^{2}+\left(\omega \varepsilon_{0} \varepsilon_{i}^{\prime}\right)^{2}} \frac{\Delta x}{S}
$$

where $S$ denotes the area where pressboard contacts with the electrodes in FDS measurement. $\varepsilon_{i}^{\prime}$ denotes the real part of complex permittivity of the $i$-th layer of pressboard. $\sigma_{i}$ represents the frequency dependent loss of $i$-th layer of pressboard and it is summation of conductive and polarization loss. $\Delta x$ is the thickness of pressboard layer and $\varepsilon_{0}$ denotes the permittivity of vacuum. Assuming that pressboard is sliced infinitely thin, (2) can be rewritten as (6)

$$
\left.Z^{*}(\omega)=\frac{1}{S}\left[\int_{0}^{l} \frac{\sigma_{x}}{\sigma_{x}^{2}+\left(\omega \varepsilon_{0} \varepsilon_{x}^{\prime}\right)^{2}} d x-j \int_{0}^{l} \frac{\omega \varepsilon_{0} \varepsilon_{x}^{\prime}}{\sigma_{x}^{2}+\left(\omega \varepsilon_{0} \varepsilon_{x}^{\prime}\right)^{2}} d x\right)\right]
$$

Once impedance $Z^{*}(\omega)$ is obtained, the complex capacitance of the pressboard can be calculated by using (7)

$$
Z^{*}(\omega)=\frac{1}{j \omega C^{*}(\omega)}=\frac{1}{j \omega C_{0} \varepsilon^{*}(\omega)}
$$

In Fig.1, each layer of pressboard can be simplified as a parallel circuit consisting of a capacitor and a resistor. For the $i$-th layer, admittance and capacitance can be written as

$$
\begin{gathered}
G_{i}^{*}(\omega)=\left(\sigma_{i}+j \omega \varepsilon_{0} \varepsilon_{i}^{\prime}\right) \frac{S}{\Delta x} \\
C_{i}^{*}(\omega)=\varepsilon_{i}^{*}(\omega) C_{0}=\varepsilon_{0} \frac{S}{\Delta x}\left(\varepsilon_{i}^{\prime}+\mathrm{j} \varepsilon_{i}^{\prime \prime}\right)
\end{gathered}
$$

Complex capacitance (8) can be expressed as (10)

$$
C_{i}^{*}(\omega)=\frac{G_{i}^{*}(\omega)}{j \omega}=\left(\varepsilon_{0} \varepsilon_{i}^{\prime}-j \frac{\sigma_{i}}{\omega}\right) \frac{S}{\Delta x}
$$

By comparing (9) and (10), it can be obtained as

$$
\begin{gathered}
\varepsilon_{x}^{\prime}=\varepsilon_{i}^{\prime} \\
\sigma_{x}=\sigma_{i}=\varepsilon_{0} \omega \varepsilon_{i}^{\prime \prime}
\end{gathered}
$$

Equations (11) and (12) reveal that $\varepsilon_{x}^{\prime}$ in (6) equals to the real part of permittivity ( $\varepsilon_{i}^{\prime}$ ) in FDS measurement while $\sigma_{x}$ in (6) can be calculated from imaginary part of permittivity $\left(\varepsilon_{i}^{\prime \prime}\right)$. Therefore, the variables $\varepsilon_{x}^{\prime}$ and $\sigma_{x}$ in (6) can be obtained from the FDS measurement when moisture is uniformly distributed.

\section{RESUlTS ANALYSIS AND EXPERIMENT VALIDATION}

This section presents detailed procedures for modelling the dielectric response of pressboard by using the proposed diffusion-driven model. Firstly, moisture distribution of pressboard is calculated. Then the dependency between permittivity and dielectric loss and diffusion position under uniform moisture distribution is explored. Afterwards, dielectric response of pressboard with non-equilibrium moisture content is calculated. Finally, modelled results are validated by laboratory experiments.

\section{A. Moisture Distribution During Diffusion Process}

During the moisture diffusion process, it is assumed that moisture penetrates from only one side of the pressboard where it is contacted with a constant source of moisture $(3.8 \%$ water content). The temperature is kept as $55{ }^{\circ} \mathrm{C}$. The pressboard is $1 \mathrm{~mm}$ thick. Finite element software is employed to calculate moisture distribution. Fig. 2 presents calculated moisture distribution in pressboard. In Fig.2, each curve represents moisture distribution along the thickness direction of pressboard with different diffusion time.

To explore a relationship between moisture concentration and permittivity as well as the dielectric loss, extensive aging experiments were conducted on oil-impregnated pressboards [14]. After moisture is uniformly distributed in the pressboard, FDS measurement ( $1 \mathrm{mHz}$ to $1 \mathrm{kHz}$ ) was performed by IDA 200 equipment. The measured real and imaginary part of permittivity of pressboards under $55^{\circ} \mathrm{C}$ with different moisture content is shown in Fig. 3 and 4 respectively.

Fig. 3 indicates that the real part of permittivity has similar decreasing trend for different moisture contents when the 
frequency increases from $1 \mathrm{mHz}$ to $1 \mathrm{kHz}$. At a particular frequency, the real part of permittivity grows up when the moisture content increases. From Fig.4 it can be seen that the hump in the imaginary part of permittivity moves toward high frequency range when moisture increase. This implies that the dielectric dissipation of pressboard is higher as more moisture content resides in the pressboard. It should be notable that in Fig. 4, some measurement errors may exist in the imaginary part at the low frequency with $1.4 \%$ moisture content. So these outlier data are not used in the following analysis part.

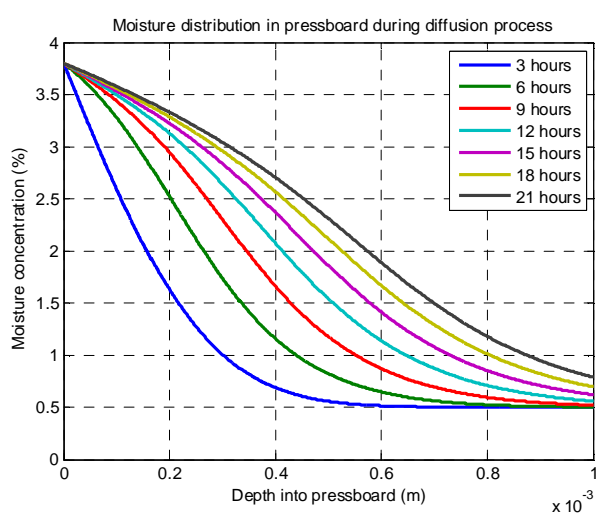

Fig. 2. Moisture distribution in pressboard during diffusion process

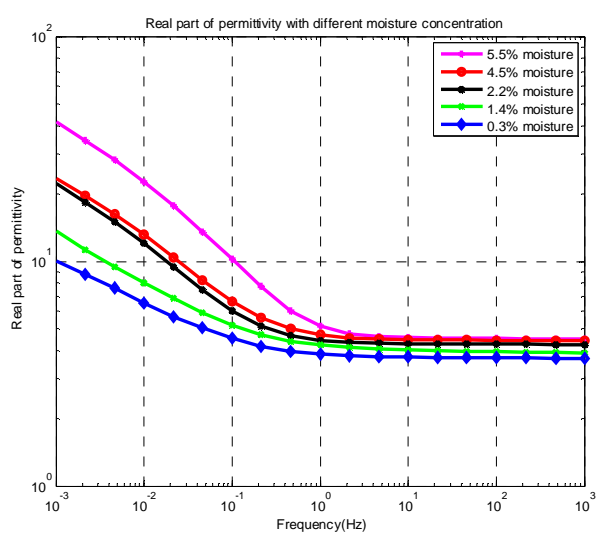

Fig. 3. Real part of permittivity under uniform moisture distribution

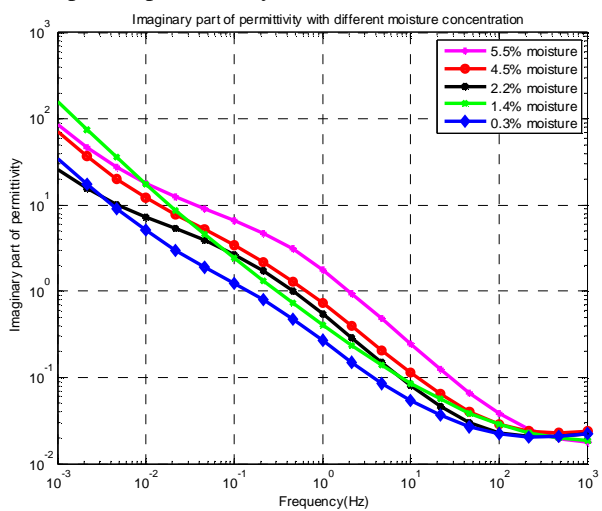

Fig. 4. Imaginary part of permittivity under uniform moisture distribution

B. Real Part of Permittivity of Oil-Impregnated Pressboard

From Fig. 3 the real part of permittivity at a particular frequency with different moisture can be obtained (points as shown in Fig.5). Suppose the real part of permittivity and the moisture concentration can be modelled as

$$
\mathcal{E}^{\prime}=e^{\alpha_{1}+\beta_{1} \cdot C}
$$

where $C$ denotes the moisture concentration. $\alpha_{1}$ and $\beta_{1}$ are parameters and computed by using least squares method. Fig.5 illustrates the fitted curve of real permittivity and Table I summarizes the fitted parameters under different frequencies. From Fig. 5 and Table I it can be seen that the real part of permittivity is exponential to the moisture concentration under each frequency. The fitted curves and measured data can be well matched.

Once the relationship between real part of permittivity and moisture content is obtained, the real permittivity $\varepsilon_{i}^{\prime}$ of the pressboard with respect to diffusion position (distance from the surface of pressboard) can be calculated by using Table I and Fig. 2. The calculated real part of permittivity and fitting curves are presented in Fig. 6 (only 18 hours diffusion is presented).

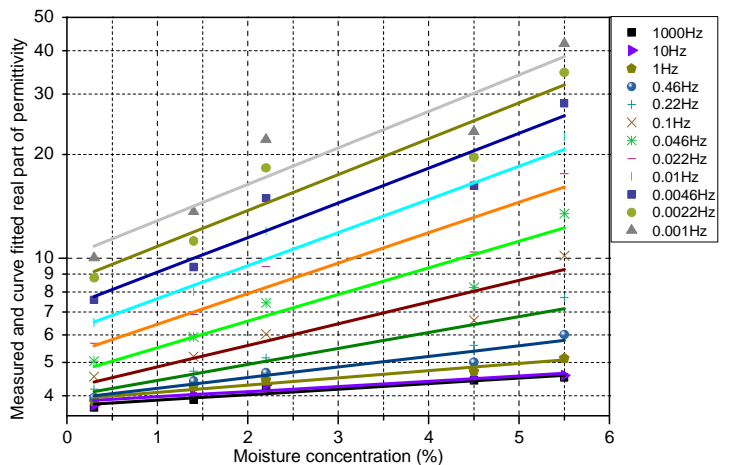

Fig. 5. Dependency between real part of permittivity and moisture under uniform distribution condition

TABLE I. FITTING PARAMETERS FOR REAL PART OF PERMITTIVITY

\begin{tabular}{|c|c|c|c|c|c|c|c|}
\hline Freq & $\boldsymbol{\alpha}_{\mathbf{1}}$ & $\boldsymbol{\beta}_{\mathbf{1}}$ & MSE & Freq & $\boldsymbol{\alpha}_{1}$ & $\boldsymbol{\beta}_{\mathbf{1}}$ & MSE \\
\hline $\mathbf{1 0 0 0}$ & 1.32 & 0.037 & 0.101 & $\mathbf{4 . 6 \times 1 0 ^ { - 2 }}$ & 1.53 & 0.178 & 1.103 \\
\hline $\mathbf{1 0}$ & 1.34 & 0.035 & 0.096 & $\mathbf{2 . 2 \times \mathbf { 1 0 } ^ { - 2 }}$ & 1.65 & 0.204 & 1.499 \\
\hline $\mathbf{1}$ & 1.36 & 0.048 & 0.101 & $\mathbf{1 0}^{-\mathbf{2}}$ & 1.81 & 0.221 & 1.980 \\
\hline $\mathbf{0 . 4 6}$ & 1.37 & 0.071 & 0.210 & $\mathbf{4 . 6 \times 1 0}^{-\mathbf{3}}$ & 1.98 & 0.232 & 2.546 \\
\hline $\mathbf{0 . 2 2}$ & 1.38 & 0.106 & 0.454 & $\mathbf{2 . 2 \times \mathbf { 1 0 } ^ { - 3 }}$ & 2.14 & 0.240 & 3.231 \\
\hline $\mathbf{0 . 1}$ & 1.43 & 0.145 & 0.765 & $\mathbf{1 0}^{-3}$ & 2.31 & 0.243 & 4.083 \\
\hline
\end{tabular}

Freq: frequency in Hz. MSE: mean square error.

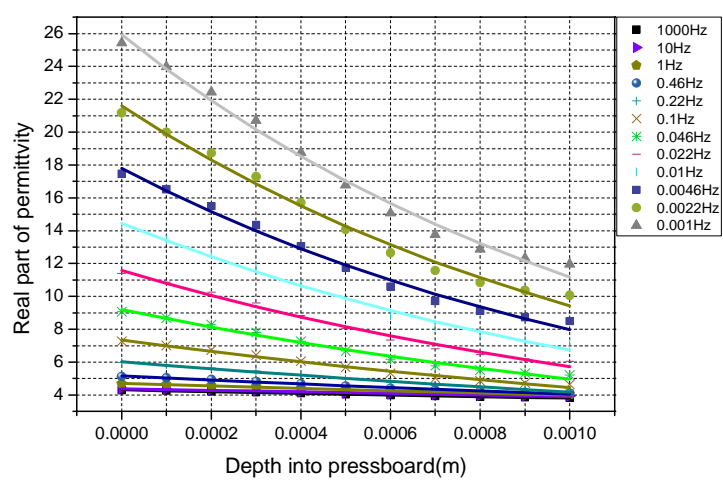

Fig. 6. Dependency between real part of permittivity and the diffusion position of pressboard (after 18 hours diffusion) 
Consider that the real part of permittivity and diffusion position has an exponential relationship

$$
\mathcal{E}^{\prime}=e^{\alpha_{2}+\beta_{2} \cdot x}
$$

where $x$ denotes position of pressboard. $\alpha_{2}$ and $\beta_{2}$ are parameters. Table II only presents the fitted parameters of (14) after 18 hours diffusion. Similarly, the real part of permittivity for the pressboard with non-equilibrium moisture distribution can be evaluated when the diffusion time varies from 3 to 21 hours.

TABLE II. FITTING PARAMETERS FOR REAL PART OF PERMITTIVITY WITH RESPECT TO DIFFUSION POSITION (AFTER 18 HOURS DIFFUSION)

\begin{tabular}{|c|c|c|c|c|c|c|c|}
\hline Freq & $\boldsymbol{\alpha}_{2}$ & $\boldsymbol{\beta}_{2}$ & Mse. & Freq & $\boldsymbol{\alpha}_{2}$ & $\boldsymbol{\beta}_{2}$ & Mse. \\
\hline $\mathbf{1 0 0 0}$ & 1.46 & -0.13 & 0.01 & $\mathbf{4 . 6 \times 1 0 ^ { - 2 }}$ & 2.22 & -0.62 & 0.04 \\
\hline $\mathbf{1 0}$ & 1.48 & -0.12 & 0.01 & $\mathbf{2 . 2 \times 1 0 ^ { - 2 }}$ & 2.45 & -0.70 & 0.06 \\
\hline $\mathbf{1}$ & 1.55 & -0.16 & 0.01 & $\mathbf{1 0}^{-\mathbf{2}}$ & 2.67 & -0.76 & 0.08 \\
\hline $\mathbf{0 . 4 6}$ & 1.64 & -0.24 & 0.01 & $\mathbf{4 . 6 \times 1 0 ^ { - 3 }}$ & 2.88 & -0.80 & 0.09 \\
\hline $\mathbf{0 . 2 2}$ & 1.79 & -0.37 & 0.02 & $\mathbf{2 . 2 \times 1 0 ^ { - 3 }}$ & 3.07 & -0.83 & 0.12 \\
\hline $\mathbf{0 . 1}$ & 1.99 & -0.50 & 0.03 & $\mathbf{1 0}^{-\mathbf{3}}$ & 3.26 & -0.84 & 0.14 \\
\hline
\end{tabular}

Freq: frequency in Hz. MSE: mean square error.

\section{Dielectric Loss of Oil-Impregnated Pressboard}

From (12) and Fig. 4, the dielectric loss of the pressboard with different moisture concentrations under a particular frequency can be plotted in Fig. 7. An exponential function is employed to describe the relationship between the loss and the moisture concentration (15)

$$
\sigma=e^{\alpha_{3}+\beta_{3} \cdot C}
$$

where $C$ denotes the moisture concentration. $\alpha_{3}$ and $\beta_{3}$ are computed by using least squares method. Table III summarizes the fitted parameters of (15). Fig.7 plots the fitted curves. From Table III and Fig.7 it can be seen that the loss and the moisture content has an exponential relationship.

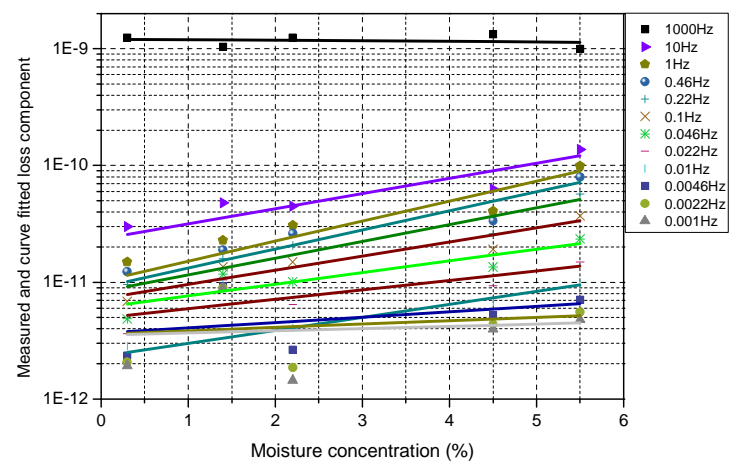

Fig. 7. Dependency between pressboard dielectric loss and moisture concentration under uniform moisture distribution condition

TABLE III. FITTING PARAMETERS FOR DIELECTRIC LOSS OF

\begin{tabular}{|c|c|c|c|c|c|c|c|}
\hline Freq & $\alpha_{3}$ & $\boldsymbol{\beta}_{3}$ & Mse. & Freq & $\boldsymbol{\alpha}_{3}$ & $\boldsymbol{\beta}_{3}$ & Mse. \\
\hline 1000 & 0.53 & -0.01 & 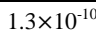 & $4.6 \times 10$ & -25.83 & 0.23 & $5 \times 10^{-12}$ \\
\hline 10 & 24.48 & 0.30 & -1 & $2.2 \times 1$ & -26.03 & 0.19 & $.3 \times 10^{-12}$ \\
\hline 1 & 25. & 0. & $1 \times 10^{-11}$ & 10 & -26 & 0.26 & $2.9 \times 1$ \\
\hline 0.46 & -25.43 & 0.38 & $8.4 \times 1$ & $4.6 x$ & -26.33 & 0.11 & $2.5 \times 10^{-12}$ \\
\hline 0.22 & 25. & 0.33 & $5.7 \times 10$ & $2.2 \times 1$ & -26 & 0.07 & $2.6 \times 10^{-12}$ \\
\hline 0.1 & -25.65 & 0.28 & $3.5 \times 10^{-12}$ & $10^{-3}$ & -26.37 & 0.04 & $2.6 \times 10^{-12}$ \\
\hline
\end{tabular}
PRESSBOARD WITH RESPECT TO MOITURE CONTENTS

Freq: frequency in Hz. MSE: mean square error
After obtaining a dependency between the dielectric loss and the moisture content of pressboard, the dielectric loss with respect to diffusion position of pressboard can also be calculated by using Fig. 2 and Table III.

The dielectric loss with respect to the diffusion position is shown in Fig. 8 and Table IV. An exponential function is used for describing the dependency between the loss and the position before uniform moisture distribution has reached

$$
\sigma=e^{\alpha_{4}+\beta_{4} \cdot x}
$$

where $x$ denotes position of pressboard. $\alpha_{4}$ and $\beta_{4}$ are computed by using least squares method.

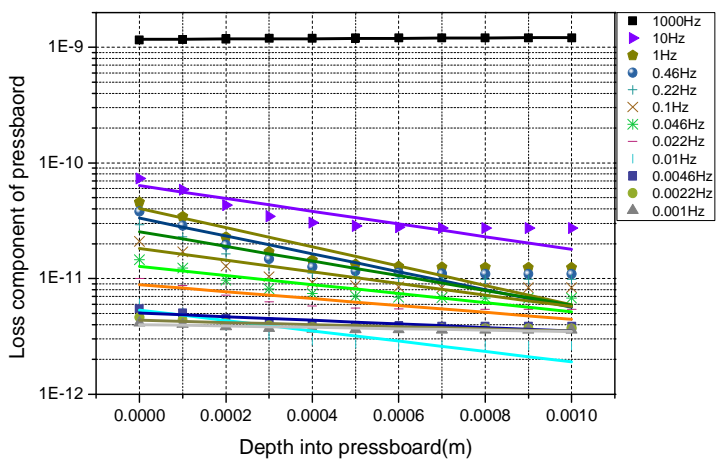

Fig. 8 Dielectric loss of pressboard along thickness direction (after 18 hours diffusion)

TABLE IV. FITTING PARAMETERS FOR DIELECTRIC LOSS WITH RESPECT

\begin{tabular}{|c|c|c|c|c|c|c|c|}
\hline Freq & $\boldsymbol{\alpha}_{4}$ & $\boldsymbol{\beta}_{\mathbf{4}}$ & Mse. & Freq & $\boldsymbol{\alpha}_{4}$ & $\boldsymbol{\beta}_{4}$ & Mse. \\
\hline $\mathbf{1 0 0 0}$ & -20.58 & 0.04 & $5.20 \times 10^{-13}$ & $\mathbf{4 . 6 \times 1 0 ^ { - 2 }}$ & -24.94 & -0.80 & $7.86 \times 10^{-14}$ \\
\hline $\mathbf{1 0}$ & -23.31 & -1.03 & $4.53 \times 10^{-13}$ & $\mathbf{2 . 2 \times 1 0 ^ { - 2 }}$ & -25.31 & -0.64 & $4.76 \times 10^{-14}$ \\
\hline $\mathbf{1}$ & -23.77 & -1.36 & $3.21 \times 10^{-13}$ & $\mathbf{1 0}^{-2}$ & -25.80 & -0.89 & $3.52 \times 10^{-14}$ \\
\hline $\mathbf{0 . 4 6}$ & -23.97 & -1.30 & $2.61 \times 10^{-13}$ & $\mathbf{4 . 6 \times 1 0}^{-3}$ & -25.92 & -0.36 & $1.76 \times 10^{-14}$ \\
\hline $\mathbf{0 . 2 2}$ & -24.23 & -1.14 & $1.90 \times 10^{-13}$ & $\mathbf{2 . 2 \times 1 0 ^ { - 3 }}$ & -26.09 & -0.23 & $1.02 \times 10^{-14}$ \\
\hline $\mathbf{1 0}^{-1}$ & -24.57 & -0.97 & $1.25 \times 10^{-13}$ & $\mathbf{1 0}^{-3}$ & -26.20 & -0.15 & $6.31 \times 10^{-15}$ \\
\hline
\end{tabular}

Freq: frequency in Hz. MSE: mean square error.

From Table II and Table IV, both real part of permittivity $\varepsilon^{\prime}$ and dielectric loss $\sigma$ of the pressboard during diffusion process can be obtained. Accordingly, the complex impedance $Z^{*}(\omega)$ of oil-impregnate pressboard after 18 hours diffusion can be calculated by solving the integral of (6). The complex permittivity $\varepsilon^{*}(\omega)=\varepsilon^{\prime}(\omega)+j \varepsilon^{\prime \prime}(\omega)$ can be calculated by (7).

\section{Moisture Diffusion Experimental Validation}

To validate the effectiveness of the proposed model, a specially designed test cell was designed and used to evaluate the dielectric response of pressboard while moisture diffusion took place [15] . During diffusion process, FDS measurement was performed on pressboards in every 3 hours.

The complex permittivity $\varepsilon^{*}(\omega)=\varepsilon^{\prime}(\omega)+j \varepsilon^{\prime \prime}(\omega)$ of pressboard with non-equilibrium moisture distribution at different diffusion time is drawn in Fig. 9 and 10. From Fig. 9 it can be observed that there is a decreasing trend in the real part of permittivity for all the circumstances. When the moisture concentration grows up, the real part of the complex permittivity will increase under each frequency. From Fig. 10 
it can be observed that the imaginary part of complex permittivity will increase within the whole frequency range when more moisture contents are in the pressboard. The increase of the imaginary part at low frequency region is more dominated. Similar to the FDS measurement under equilibrium condition, the dielectric relaxation centres also move from lower frequency region to higher frequency band. This implies that the dielectric loss of humid pressboard is large than that of a drier one.

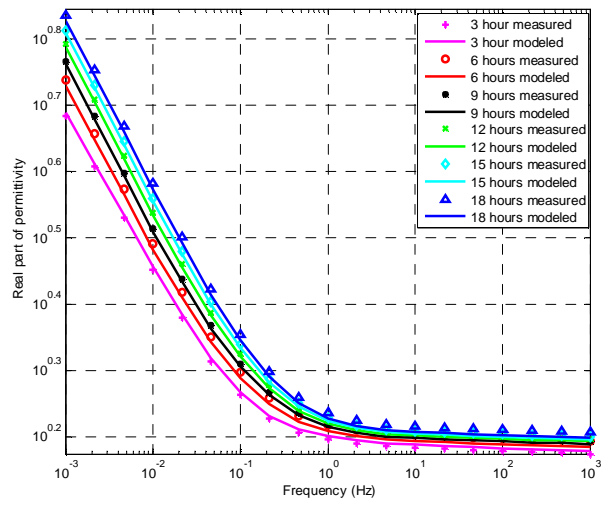

Fig. 9. Comparison between measured and calculated real part of complex permittivity

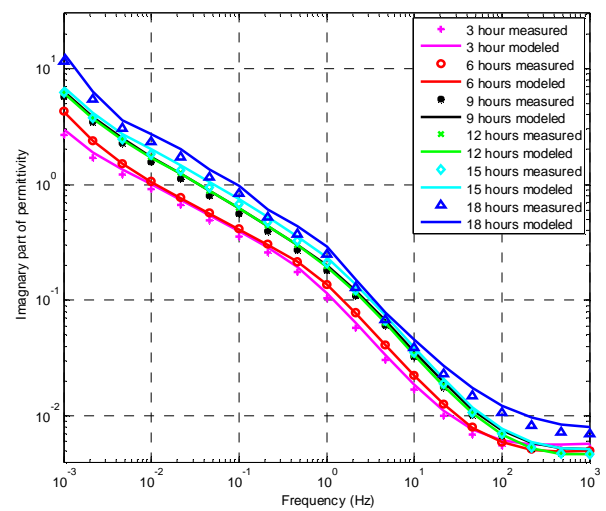

Fig. 10. Comparison between measured and calculated imaginary part of complex permittivity

The possible diffusion mechanism can be elaborated as follows. When the moisture diffusion starts, dipoles of water molecules are non-homogeneously distributed in the pressboard. Once an external electric field is applied, more energy will be consumed to orient the moisture dipoles in the humid part of the pressboard. At the same time, the dipoles of water molecular will establish an asymmetric additional electric field. The residual free charges (electrons or ions) in the pressboard need to overcome not only the local electric potential barrier but also the additional field caused by the polarized moisture dipoles. Although the pressboard can reach $3.8 \%$ moisture level at the location it contacts the moisture source, the other side of pressboard only reaches $0.75 \%$ moisture level after 18 hours of diffusion. As the charges keep moving away from the diffusion surface of the pressboard, less charges are available in the drier part of the pressboard. Therefore, it becomes more difficult for these charges to pass through the remaining of pressboard and this may result in the reduction in the dielectric loss.

\section{CONCLUSIONS}

This paper proposed a diffusion-driven model for evaluating the effect of moisture diffusion on the dielectric response measurement in a transformer. Based on the proposed model, dielectric relaxation behaviour of pressboard due to the moisture migration between oil and paper was studied. Dielectric response measurement (e.g. FDS) is performed on pressboards and results are used to validate the proposed model. It is expected that this model can be applied for assessing the insulation condition of the transformer under moisture disequilibrium condition.

\section{ACKNOWLEDGMENT}

We highly appreciate the supports provided by Australian Research Council, Powerlink Queensland, Energex, Ergon Energy, and TransGrid. The authors also would like to thank Raj B. Jadav for providing experimental results to validate the model.

\section{REFERENCES}

[1] D. F. Garcia, R. Villarroel, B. Garcia and J. Burgos, "A review of moisture diffusion coefficients in transformer solid insulation - Part 2 : Experimental validation of the coefficients," IEEE Electr. Insul. Mag., vol.29, pp. 40-49, 2013.

[2] D. F. Garcia, B. Garcia and J. Burgos, "A review of moisture diffusion coefficients in transformer solid insulation-part 1: Coefficients for paper and pressboard," IEEE Electr. Insul. Mag., vol.29, pp. 46-54, 2013.

[3] D. F. Garcia, B. Garcia, J. C. Burgos and N. G. Hernando, "Experimental determination of the diffusion coefficient of water in transformer solid insulation," IEEE Trans. Dielectr. Electr. Insul., vol.19, pp. 427-433, 2012.

[4] B. Garcia, J. C. Burgos, A. M. Alonso and J. Sanz, "A moisture-in-oil model for power transformer monitoring - Part II: Experimental verification," IEEE Trans. Power Del., vol.20, pp. 1423-1429, 2005

[5] B. Garcia, J. C. Burgos, A. M. Alonso and J. Sanz, "A moisture-in-oil model for power transformer monitoring - Part I: Theoretical foundation," IEEE Trans. Power Del., vol.20, pp. 1417-1422, 2005.

[6] Y. Du, A. V. Mamishev, B. C. Lesieutre and M. Zahn, "Measurement of moisture diffusion as a function of temperature and moisture concentration in transformer pressboard," in Proceedings of 1998. Annual Report of Conference on Electrical Insulation and Dielectric Phenomena, 1998,Atlanta, GA, pp. 341-344.

[7] W. Guidi and H. Fullerton, "Mathematical methods for prediction of moisture take-up and removal in large power transformers," in Proceedings of IEEE Winter Power Meeting, 1974,242-244.

[8] P. Ast, "Movement of moisture through a50p281 kraft paper (dry and oil impregnated),", General Electric, Test Report HV-ER-66-41, 1966.

[9] D. F. García, B. García, J. C. Burgos and N. García-Hernando, "Determination of moisture diffusion coefficient in transformer paper using thermogravimetric analysis," International Journal of Heat and Mass Transfer, vol.55, pp. 1066-1075, 2012.

[10] R. Neimanis, "On estimation of moisture content in mass impregnated distribution cables," Ph.D dissertation, KTH, Sweden, 2001.

[11] S. D. Foss and L. Savio, "Mathematical and experimental analysis of the field drying of power transformer insulation," IEEE Trans. Power Del., vol.8, pp. 1820-1828, 1993.

[12] A. Howe, "Diffusion of moisture through power-transformer insulation," in Proceedings of Institution of Electrical Engineers, 1978,978-986.

[13] C. Ekanayake, S. M. Gubanski, A. Graczkowski and K. Walczak, "Frequency response of oil impregnated pressboard and paper samples for estimating moisture in transformer insulation," IEEE Trans. Power Del., vol.21, pp. 1309-1317, 2006.

[14] R. B. Jadav, T. K. Saha and C. Ekanayake, "Understanding moisture diffusion process in oil-impregnated pressboard insulation of transformer," in Proceedings of 2011 IEEE Power and Energy Society General Meeting, 2011,San Diego, CA, pp. 1-8. 